High Energy Facilities

RHIC-15

Advanced Projects

BROOKHAVEN NATIONAL LABORATORY

Associated Universities, Inc.

Upton, New York 11973

RHIC Technical Note No. 15

Possible Benefits from Shuffling Dipoles in the RHIC

S. Ohnuma*

December 10, 1985

*Fermi National Accelerator Laboratory, P.0. Box 500, Batavia, IL 60510 


\title{
Possible Benefits from Shuffling Dipoles in the RHIC
}

\author{
S. Ohnuma* \\ December 10, 1985
}

*

Fermi National Accelerator Laboratory, P.0.Box 500, Batavia, IL 60510 


\section{Introduction}

It is essential to state at the outset that there is no unique way of shuffling magnets. Many factors are involved in deciding how to do it; . for example, one may take into account not just the linear machine parmeters but other things such as size and distribution of magnet errors, magnet installation schedule, allowance (or non-allowance) of "unusable" magnets and type and scope of diagonostic systems and correction systems. In addition, one may be influenced, conciously or unconciously, by the past experiences and may be inclined to emphasize some factors over others even when that is not justified by technical considerations alone. The example given in this. note is just that, an example of what one can do under certain assumptions. Better ways of shuffling magnets should emerge as more data on field qualities would become available.

For the Tevatron at Fermilab, the goal of shuffling dipoles was a quite limited one and, because of that, the problem was a well-defined one. 1 we simply tried to minimize the magnitude of several isolated resonance-driving terms, these resonances arising from sextupole $\left(b_{2}\right.$ and $\left.a_{2}\right)$ and octupole $\left(a_{3}\right.$ only) components. The dimensionless figure-of-merit was the magnitude of each term relative to what one should expect from the distribution of $b_{2}, a_{2}$ or $a_{3}$ if the shuffling were not done. Since this involves only one particular harmonic component for each resonance, it is the simplest case of what one might call the "global" compensation. ${ }^{2}$ (The nature of "global" and "local" compensations will be explained below.) Another example of the global compensation has been discussed recently ${ }^{3}$ in which many harmonic components near the most important one are minimized by a particular way of shuffiing. This sort of consideration becomes necessary when one is concerned about the loss of linearity in the beam motion, which may cause a reduction in the dynamic aperture of the machine, even though isolated resonances are not a direct threat to the beam stability.

In contrast to the global compensation, the "local" compensation is more appropriate when the source of field errors (or nonlinear elements) is within a relatively. small area of the ring. One then tries to confine the effect of 
errors within that area. If the compensation is perfect, there wil1 be no effect outside the area although the effect may not be so smal1 inside. This scheme has been promoted especially by Tom Collins ${ }^{4}$ in connection with a group of special sextupoles in the SSC lattice. The difference in approach between two compensation.schemes, global and local, can be seen, - for example, in two different (but completely equivalent) forms for $\Delta \beta / \beta$, the error in betatron amplitude function $\beta$ caused by the quadrupole component $b_{1}$ in dipoles:

a) global

$$
\begin{aligned}
& (\Delta \beta / \beta) \text { at } \psi=-(\nu / \pi) \sum_{n=-\infty}^{\infty} \frac{J_{n}}{4 \nu^{2}-n^{2}} e^{i n \psi / \nu} \\
& \text { with } J_{n}=\sum_{k=1}^{M}\left(\beta \theta b_{1}\right)_{k} e^{-i n \psi_{k} / \nu} \\
& \psi=\text { betatron phase, } \theta=\text { bend angle, } \nu=\text { tune. }
\end{aligned}
$$

Eq. (1) is valid at any location around the ring so that the source of error $b_{\gamma}, \quad k=1$ to $M$ magnets, can be distributed a 11 around the ring. Obvious $1 y$, one tries to minimize $J_{n}{ }^{\prime} s$ with $n$ near (2v).

\section{b) $\underline{\text { local }}$}

Here the source of error $b_{1}$ is confined to a small area. The goal is to minimize or completely eliminate $(\Delta \beta / \beta)$ at all points outside this area. For this, one must consider $\Delta \alpha$ together with $(\Delta \beta / \beta)$. Consider an arbitrary point outside and take this point as the origin of phase $\psi$. We then have

$$
(\Delta \beta / \beta)-i\left(\Delta \alpha-\frac{\alpha}{\beta} \Delta \beta\right)=\frac{-e^{2 i \pi \nu}}{2 \sin (2 \pi \nu)} \sum_{k=1}^{M}\left(\beta \theta b_{1}\right)_{k} e^{2 i \psi_{k}}
$$

If $M$ magnets are arranged such that the summation in Eq.(3) is zero, the errors $\Delta \alpha$ and $(\Delta \beta / \beta)$ are zero everywhere outside the $M$ magnets. 
It should be noted here that, inside the region under consideration, $\Delta \alpha$ and $(\Delta \beta / \beta)$ could be large. If the error is dipole field $b_{0}$ or $a_{0}$ instead of the quadrupole field $b_{1}$, the effect will be on the horizontal or vertical dispersion. An interesting example of this is the overpass at $B \emptyset$ of the main ring at Fermilab. ${ }^{5}$ The beam line is raised by $19^{\prime}$ near $B \emptyset$ in such a way that the vertical dispersion around the ring outside the overpass area is minimized to less then $0.5 \mathrm{~m}$ but it is as large as $5 \mathrm{~m}$ inside the overpass.

\section{Special Considerations for the RHIC}

One obvious difference between the RHIC and the Tevatron is in the number of dipoles, 144 in the regular arc sections of the RHIC compared with almost 800 for the Tevatron. Calculations which we regarded as impractical because of the required computing time for the Tevatron may not be so for the RHIC. Another difference (which may be more relevant to the shuffling) is that, for the Tevatron, the fluctuations in quadrupole components $b_{1}$ and $a_{1}$ were reduced down to $0.5 \times 10^{-4}$ (at $1^{11}$, rms) by moving the collared coil relative to the surrounding yoke. Since the effect of $\left(b_{j}, a_{1}\right)$ was negligible, we concentrated on minimizing the effects of nonlinear field components. For the RHIC, the situation seems to be the other way around; the linear effects due to $b_{1}$ and $a_{1}$ on betatron amplitudes and dispersions may reduce the effective aperture of the ring more than noninear effects arising from higher multipole components such as $b_{2}$ and $a_{2}$. Therefore, it is assumed here that

(i) In shuffling dipoles in the regular arc sections, only the effects of $b_{1}$ on $\beta_{x}, \beta_{y}$ and $x_{p}$ (horizontal dispersion), and the effects of $a_{1}$ on $Y_{p}$ (vertical dispersion) are taken into account. The effect of $b_{2}$ is controlled only to the extent that it is no more than one would expect from statistical arguments.

The choice of the number of dipoles to be shuffled each time will undoubtedly depend on the schedule of magnet construction and tunnel preparation. It may even change during the course of the project as it did for 
the Tevatron. Here we take eight or twelve as a reasonable choice covering four or six regular cells. With less than eight magnets, it will be difficult to balance the errors (particularly when some errors are abnormal) while more than six cells would cover too much phase advance.

(ii) Two cases are considered, one with eight dipoles and the other with twelve in each group to be shuffled.

Problems associated with magnet errors in the insertions are rather special. They may be compensated for by special shunts or separate power supplies. Even if it becomes necessary to shuffle insertion magnets, it should be done independently from the shuffling of regular dipoles. It is expected that the effect of errors in regular quadrupoles is much less than that of dipole errors. Again, any shuffling of quadrupoles should be done separately.

(iii) All regular quadrupoles are assumed to be free of errors. Insertions are assumed to be perfect.

\section{Calculations for Shuffling}

Since the purpose of this note is simply to demonstrate how shuffling can be done to minimize various effects of magnet errors, a precise quantitative estimate of these effects is not an essential requirement. In order to simplify the computation, all magnets (quadrupoles and dipoles) in the arc sections are treated as a thin 1ens. Moreover, each insertion is represented by a matrix that matches a11 linear parameters with the phase advance of $636^{\circ}$ in both directions. The cell length is $29.622 \mathrm{~m}$ and the bend angle is $38.85 \mathrm{mr}$ per dipole. Shufflings are done for $\nu_{x}=\nu_{y}=28.8$ corresponding to phase advance of $91^{\circ} /$ cel1 but the performance is checked for $v_{x}=v_{y}=28.4$ to see that it is not degraded by a small change in tune.

* The most important error in regular quadrupoles is the fluctuation in the integrated gradient field. It may be difficult to shuffle quadrupoles unless one is certain of the average over the entire ring. At the same time, it does not seem practical to postpone the installation untill all of them are built and measured. 
The distribution of $b_{7}, a_{1}$ and $b_{2}$ is all taken to be Gaussian around the mean zero with the rms values

$$
\left\langle b_{1}\right\rangle=2.1 \times 10^{-4} / 25 \mathrm{~mm}, \quad\left\langle a_{1}\right\rangle=4.3 \times 10^{-4} / 25 \mathrm{~mm}, \quad\left\langle b_{2}\right\rangle=4.6 \times 10^{-4} /(25 \mathrm{~mm})^{2}
$$

Using these numbers, one can estimate the expected value of various errors due to 144 dipoles:

$$
\begin{aligned}
& \left\langle\frac{\Delta \dot{\beta}}{\beta}\right\rangle_{x},\left\langle\frac{\Delta \beta}{\beta}\right\rangle_{y}=\frac{1}{2 \sin \mid 2 \pi v T .} \frac{1}{\sqrt{2}}<b_{1}>\theta_{B} \beta_{x}{ }^{\prime}(144)^{\frac{1}{2}}=0.0322 \text {, } \\
& \left\langle\Delta x_{p} / \sqrt{ } \beta_{x}\right\rangle=\frac{1}{2 \sin |\pi \nu|} \frac{1}{\sqrt{ } 2}<b_{1}>\theta_{B} x_{p} \beta_{x}(144)^{\frac{1}{2}}=0.0109 m^{\frac{1}{2}}, \\
& \left\langle\gamma_{p} / \sqrt{ } \beta_{y}>=\frac{1}{2 \sin |\pi \nu|} \frac{1}{\sqrt{ } 2}<a_{1}>\theta_{B} x_{p} \beta_{y}(144)^{\frac{1}{2}}=0.0224 m^{\frac{1}{2}}\right.
\end{aligned}
$$

where, on the right hand side of each equation, $\theta_{B}=0.03885$ (bend angle), $\beta_{x}=\beta_{y}=22.7 \mathrm{~m}$ and $X_{p}=0.99 \mathrm{~m}$ at each dipole (regarded as a thin lens). As the measure of deviations from linearlity in betatron oscillations, we use the distortion functions $\left(B_{3}^{2}+A_{3}^{2}\right)^{\frac{1}{2}},\left(B_{s}^{2}+A_{s}^{2}\right)^{\frac{1}{2}}$ and $\left(B_{d}^{2}+A_{d}^{2}\right)^{\frac{1}{2}}$ defined by Tom Collins. ${ }^{4}$ There are two more pairs of functions, $\bar{B}$ and $\bar{A}$, and $B_{1}$ and $A_{1}$ but their expected values are not much different from that of $\left(B_{d}^{2}+A_{d}^{2}\right)^{\frac{1}{2}}$. Expected values are, for $v=28.8$,

$$
\begin{aligned}
& \left.<\left(B_{3}^{2}+A_{3}^{2}\right)^{\frac{1}{2}}\right\rangle=\frac{1}{16} \frac{1}{\sin \mid 3 \pi v_{x}}<b_{2}>\theta_{B}\left(\beta_{x}^{3} / \beta_{0}\right)^{\frac{1}{2}}(144)^{\frac{1}{2}}=2.34 m^{-1}, \\
& \left.<\left(B_{S}^{2}+A_{s}^{2}\right)^{\frac{1}{2}}\right\rangle=\frac{1}{16} \frac{1}{\sin \left|\pi\left(\nu_{x}+2 \nu_{y}\right)\right|}<b_{2}>\theta_{B}\left(\beta_{x} B_{y}^{2} / \beta_{0}\right)^{\frac{1}{2}}(144)^{\frac{1}{2}}=2.34 m^{-1}, \\
& \left.<\left(B_{d}^{2}+A_{d}^{2}\right)^{\frac{1}{2}}\right\rangle=\frac{1}{16} \frac{1}{\sin \mid \pi\left(\nu_{x}-2 \nu_{y}\right) T}<b_{2}>\theta_{B}\left(\beta_{x} B_{y}^{2} / \beta_{0}\right)^{\frac{1}{2}}(144)^{\frac{1}{2}}=3.79 m^{-1}
\end{aligned}
$$

where the reference value of $\beta$ is taken to be $\beta_{0}=7 \mathrm{~m}$. 
One random set of $\left(b_{1}, a_{1}, b_{2}\right)$ was generated for 144 dipoles and the calculations were always made for this particular set. The comparison is made between the unique, optimally shuffled arrangement of this set and 1,000 randomiy arranged rings using the same set of $\left(b_{1}, a_{1}, b_{2}\right)$. As the figureof-merit, a simpie expression

$$
\text { F.M. } \begin{aligned}
\equiv\left|\Sigma a_{1} \exp \left(i \psi_{y}\right)\right|^{2} & +\left|\Sigma b_{1} \exp \left(i \psi_{x}\right)\right|^{2}+\left|\Sigma b_{1} \exp \left(2 i \psi_{x}\right)\right|^{2} \\
& +\left|\Sigma b_{1} \exp \left(2 i \psi_{y}\right)\right|^{2}
\end{aligned}
$$

evaluated at dipole locations was initially used with the supplementary condition that

$$
\left|\Sigma b_{2} \exp \left(3 i \psi_{x}\right)\right| \&\left|\Sigma b_{2} \exp \left(i \psi_{ \pm}\right)\right| \quad\left(\psi_{ \pm} \equiv \psi_{x} \pm 2 \psi_{y}\right)
$$

do not exceed the expected rms values. The summations here are over eight or twelve dipoles of each group so that one is trying to minimize the effect of each group outside the four or six cells under consideration. In shuffling magnets in the second group, it might be better to include the predetermined sums over the first group. Then for the third shuffling, the sums would include the results from the two previous groups, and so on. However, this is not necessarily the optimum procedure since the "inside" region in which the minimization is not done at all covers larger and larger fraction of the. entire ring. For the best overall result, it is not obvious what the largest number of groups should be in the summation. It was then realized that, for a given arrangement of al1 magnets, linear lattice parameters $\left(\beta_{x}, \beta_{y}, X_{p}, Y_{p}\right)$ can be calculated rapidly at all locations around the ring so that the figureof-merit could be more directly related to these parameters. Results presented in the next section have been obtained with the figure-of-merit

$$
\text { F.M. } \equiv \Sigma\left(\Delta \beta_{x} / \beta_{x}\right)^{2}+\Sigma\left(\Delta \beta_{y} / \beta_{y}\right)^{2}+\Sigma\left(\Delta x_{p} / \sqrt{ } \beta_{x}\right)^{2}+\Sigma\left(Y_{p} / \sqrt{ } \beta_{y}\right)^{2}
$$

where the summations are at al1 (6×25) quadrupole locations, "inside" as well as "outside" regions. Each quantity to be summed is calculated exactly for a given arrangement of dipoles with $M$ dipoles, $2 M$ dipoles, $3 M$ dipoles, 
and so on where $M=8$ or 12 . For shuffling the last $M$ dipoles, (144-M) dipoles are already determined and the ring is entirely "inside". The local nature of balancing is thus shifted gradualiy to the global nature. In order to find the final "optimum" arrangement, approximately 1,000 random cases were studied. Although the figure-of-merit does not include the effect of sextupole component $b_{2}$, quantities such as $\left(B_{3}^{2}+A_{3}^{2}\right)$ summed at a11 150 quadrupole locations were monitored to prevent large nonlinear effect in the selected "optimum" arrangement. It is of course possible to add nonlinear. distortion effects arising from the skew sextupole component $a_{2}$ for this monitoring as long as one is not too greedy.

\section{Results}

Seven quantities, four of them linear and three nonlinear, are calculated to test the performance of the shuffiing.
I. $\frac{1}{\sqrt{7} 50}\left\{\Sigma\left(\Delta \beta_{x}^{*} / \beta_{x}\right)^{2 \frac{1}{2}}\right.$
II. $\frac{1}{\sqrt{150}}\left\{\Sigma\left(\Delta \beta_{y} / \beta_{y}\right)^{2}\right\}^{\frac{1}{2}}$

III. $\frac{1}{\sqrt{150}}\left\{\Sigma\left(\Delta X_{p} / \sqrt{ } \beta_{x}\right)^{2}\right\}^{\frac{1}{2}}$

IV. $\frac{1}{\sqrt{150}}\left\{\Sigma\left(Y_{p} / \sqrt{ } \beta_{y}\right)^{2}\right\}^{\frac{1}{2}}$

v. $\frac{1}{\sqrt{150}}\left\{\Sigma\left(B_{3}^{2}+A_{3}^{2}\right)\right\}^{\frac{1}{2}}$

V. $\frac{1}{\sqrt{150}}\left\{\Sigma\left(B_{S}^{2}+A_{S}^{2}\right)\right\}^{\frac{1}{2}}$

VII. $\frac{1}{\sqrt{150}}\left\{\Sigma\left(B_{d}^{2}+A_{d}^{2}\right)\right\}^{\frac{3}{2}} \quad$ (Summations are over 150 quadrupole locations.)

The shuffled arrangement is compared with 1,000 randomly arranged cases with eight or twelve dipoles as a unit. The tune used to find the optimum arrangement is 28.8 in both horizontal and vertical directions $191^{\circ} /$ regular ce11) but the same arrangenient is used with the tune of $28.4\left(89^{\circ} /\right.$ regular ce11) to see the tune dependence of the performance. In comparing the performance, the "rank" of 0 means the shuffled case is better than any of 1,000 cases and 1,000 means worse than any. 
Table 1. $\quad M=8$ (eight dipoles shuffled each time); $\quad \nu=28.8$

\begin{tabular}{|c|c|c|c|c|c|c|}
\hline & expected & \multicolumn{2}{|c|}{$\begin{array}{l}\text { average of } \\
1,000 \text { cases }\end{array}$} & $\begin{array}{l}\text { 1argest of } \\
1,000 \text { cases }\end{array}$ & $\begin{array}{l}\text { shuffled } \\
\text { arrangement }\end{array}$ & rank \\
\hline I. & 0.0322 & \multicolumn{2}{|c|}{0.0330} & 0.0742 & 0.0074 & 0 \\
\hline II. & 0.0322 & \multicolumn{2}{|c|}{0.0319} & 0.0676 & 0.0069 & 0 \\
\hline III. & 0.0109 & \multicolumn{2}{|c|}{0.0115} & $0.029\}$ & 0.0021 & 0 \\
\hline IV. & 0.0224 & \multicolumn{2}{|c|}{0.0248} & 0.0619 & 0.0044 & 0 \\
\hline v. & 2.34 & \multicolumn{2}{|c|}{2.31} & 4.59 & 1.69 & 221 \\
\hline VI. & 2.34 & \multicolumn{2}{|c|}{2.39} & 5.10 & 1.97 & 365 \\
\hline VII. & 3.79 & \multicolumn{2}{|c|}{3.48} & 8.51 & 1.14 & 8 \\
\hline \multicolumn{7}{|c|}{$M=8 ; \quad v=28.4$} \\
\hline & fled arra & ement & $\begin{array}{r}\text { I. } \\
\text { II. } \\
\text { III. } \\
\text { IV. } \\
V . \\
V I . \\
\text { VII. }\end{array}$ & $\begin{array}{l}0.0140 \\
0.0063 \\
0.0019 \\
0.0108 \\
3.27 \\
3.22 \quad \text { * } \\
1.20\end{array}$ & 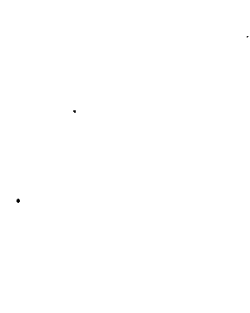 & \\
\hline
\end{tabular}


Table 2. $\quad M=12$ (twelve dipoles shuffled each time); $\quad v=28.8$

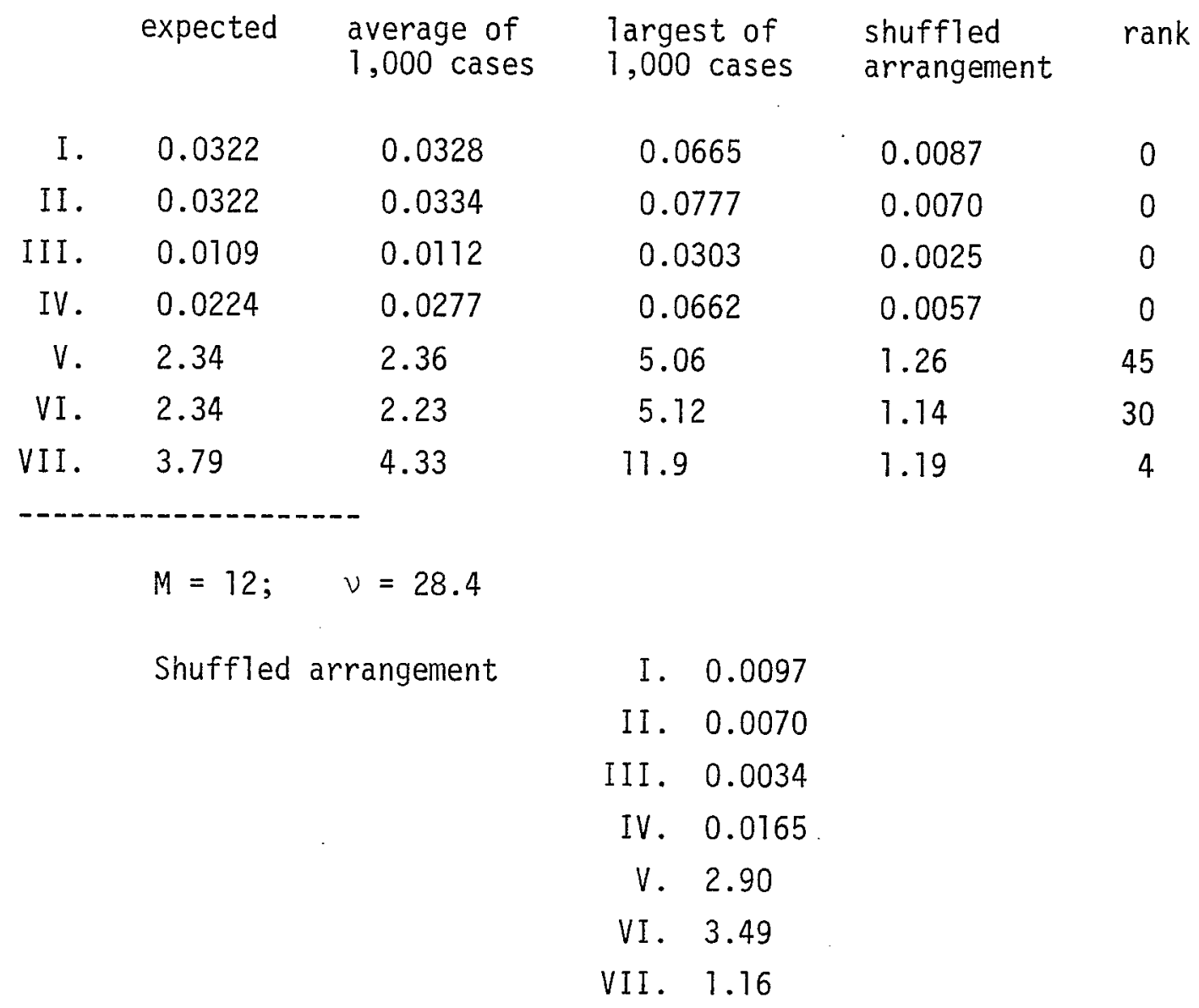

Conclusion

With the Gaussian distribution, it seems possible to achieve an improvement of factor four to five over the statisticaliy expected values without too much sacrifice in the nontinear distortion. There is no difference in the performance between $M=8$ and $M=12$ and the tune dependence of the performance is acceptable when the change in tune is less than $\sim 0.5$. 


\section{References}

1. IEEE Trans. Nucl. Sci., NS-30 (1983), p. 2472.

2. Intersection. Between Particle and Nuclear Physics, AIP Conference Proceedings No. 123 (1984), p. 415.

3. IEEE Trans. Nucl. Sci., NS-32 (1985), p. 2314.

4. Tom Collins, "Distortion Functions", Fermilab-84/114, October 23, 1984.

5. TM-1124, Fermilab, August 10, 1982. 